\section{Laboratory diagnosis of malaria}

The article by Warhurst et al raised interesting practical issues regarding the laboratory diagnosis of malaria, which are often missed by many inexperienced parasitologists and technical staff. Aspects of storage in relation to time, type of anticoagulant, and subsequent interpretation certainly needs careful consideration before making a diagnosis Further to Warhurst et al's article, we briefly discuss our experience of the detection of malaria parasite in routine diagnosis.

In an ongoing prospective study, we are evaluating the sensitivity of acridine orange staining compared to techniques such as quantitative buffy coat, Giemsa stain, Zenner stain, Field's stain, and HRP-II antigen detection.

Diagnosis of malaria using acridine orange staining has been performed since initia studies reported lower sensitivity and specificity than Giemsa. ${ }^{2}$ Recently, however, acridine orange staining using interference filter in a light microscope has shown four times more sensitivity than the conventional Giemsa stain. ${ }^{3}$ All of the test smears (244) and contro smears (50) in our study have been examined by both acridine orange and Giemsa staining. All slides were coded in a blind manner and were examined separately by two groups of observers, and confirmed by a senior parasitologist. All the developmental stages of parasite were easily identified at a magnification of $\times 900$ under special oil immersion lens (Paralens, Becton Dickinson, USA). The time required to find the first parasite in these smears ranged from five seconds to five minutes. We also evaluated acridine orange and Giemsa in relation to stages and density of parasite found. Parasite count was 1.5 times higher using acridine orange than using Giemsa. In only two test samples asexual stages of Plasmodium falciparum were found using acridine orange staining with parasite count less than $100 / \mu$ l of blood that could not be detected by Giemsa.

Acridine orange staining is also cheaper and faster than quantitative buffy coa examination. Morphological findings were much clearer using acridine orange compared with quantitative buffy coat examination (as the concentration of parasite is very high in buffy layer). We strongly support the continued use of Giemsa stain in laboratory diagnosis of malaria; however, we find acridine orange staining more reliable and consistent. It can show positivity with much lower parasitaemia compared with other stains One question which we are not yet able to answer is how long acridine orange stained slides can be kept for referral or review.

B R MIRDHA J C SAMANTARAY B MISHRA

All India Institute of Medical Sciences Ansari Nagar New Delhi 110029 India

1 Warhurst DC, Williams JE. Laboratory diagnosis of malaria. $\mathcal{F}$ Clin Pathol 1996;49:533-8.

2 Shute AT, Sodeman TM. Identification of malaria parasites by fluorescence microscopy and acridine orange staining. WHO Bull 1973; 48:591-6.

3 Agbani Hind $\mathrm{MM}, \mathrm{El}$ Has Imad Abdain, El Town Islam Abdalla, El Hassan AM. FluoresTown Islam Abdalla, El Hassan AM. Fluoresfille with ascopy using a light microscope sis of malaria. Trans Roy Soc Trop Med Hyg sis of malaria.

\section{Book reviews}

Slide Atlas of Fungal Infection. Three volumes. Richardson MD, Warnock DW, Campbell CK. (£195.00.) Blackwell Science. 1995. ISBN 0865428964 .

The management of fungal infections has, until recent years, been largely the province of dermatologists and mycologists, with the result that there has been considerable ignorance about fungi, even among microbiologists. Richardson and Warnock have made great efforts to address this problem over the past decade, publishing, among others, two important clinical books. Together with Campbell, they have produced this slide atlas to complement one of these (Fungal Infection-Diagnosis and Management; Blackwell Science, 1993).

The atlas is presented in three sets covering superficial fungal infections, systemic fungal infections, and subcutaneous and unusual fungal infections. There are 150 slides in all. Each set comes with a slim volume of notes containing a black and white photograph of each slide and a few accompanying sentences.

The content is excellent. The authors have assembled a nicely balanced mixture of photographs of clinical cases, histological slides, culture plates, and fungal micrographs. To be truly comprehensive it would be necessary for each organism (with the exception of the few non-culturable ones) to be illustrated by at least one each of these. However, this would undoubtedly have added considerably to the cost and size of the atlas and the authors appear to have made a conscious decision to be selective.

The publishers suggest that the atlas can be used as a diagnostic aid. Mycologists and microbiologists might well wish to use the booklets for this purpose, but their value would have been greatly enhanced if the illustrations had been in colour.

This atlas will make good material for the teaching of undergraduates, postgraduates, laboratory staff and clinicians. I recommend it to all microbiology, histology, and infectious diseases departments.

C KIBBLER

Current Clinical Topics in Infectious Diseases. Remington J, Swartz M, eds. (Pp 317; £57.50.) Blackwell Science. 1996. ISBN 0865424772.

The ever escalating pace of medical publishing has resulted in the emergence of a number of excellent literature review journals in the field of medical microbiology and infectious diseases. These enable the busy practitioner to keep up to date with key papers but they also emphasise the speed with which textbooks become outdated. Current clinical topics is one book which aims to fill the textbook gap. It has been published annually since 1980 and has reviewed a host of timely topics.

The present volume, number 16 in the series, comprises (like previous volumes) a collection of topics selected by the editors, and written and published within a year of commissioning. Three of the 14 chapters review antibiotic resistance in different organisms, reflecting the current anxieties in the infectious diseases' world. Other chapters cover community and hospital acquired pneumonia, toxic shock syndrome, HIV-2, infections of the eyelid, lacrimal system, conjunctive and cornea, bacterial meningitis, the pathogenesis of tuberculosis, Vibrio cholerae 0139, ehrlichiosis, cystercercosis, and invasive aspergillosis.

Some authors have aimed at a short update while others have delivered a more comprehensive overview. This is also reflected in the use of tables and illustrations (both absent in some chapters). Most chapters are well referenced, some authors citing more than 300 references. All are up to date, several using references from 1995. I would select the chapters by Thomson et al on novel plasmid mediated $\beta$ lactamases in Enterobacteriaceae, and by D'Aquila on HIV-2 as being particularly good updates for those whose microbiological interests lie in other fields; Denning's use of illustrations demonstrates well the role of CT scanning in the diagnosis of invasive aspergillosis.

There is something here for everyone and the anticipated readership seems to include both trainees and trainers, whether microbiologists or infectious disease clinicians. The series certainly shows no signs of flagging.

C KIBBLER

\section{Gastrointestinal Mucosal Biopsy.} Goldman H. (Pp 608, 509 halftones, 26 colour plates; US\$95.00.) Churchill Livingstone. 1996. ISBN 0443089906.

The idea of a single volume dealing with mucosal biopsy of the whole gastrointestinal tract has always appealed to me, and so it was with eager anticipation that I started to read this new book. Of course it would have to be pretty special to surpass or even equal the tried and tested Biopsy pathology series, but I was willing to give it a try. Quite rapidly, however, eager anticipation was replaced by frustrated disappointment as I realised it fell well short of the mark.

The book is volume 20 in the American series Contemporary issues in surgical pathology. It deals with all sites in the gastrointestinal tract from oesophagus to anal canal in $\mathbf{5 7 0}$ medium-sized pages. Like most large textbooks of gastrointestinal pathology, a functional approach is adopted; individual disease entities are classified and described and their histological features listed. Unfortunately, this approach does not work in a text on mucosal biopsies. The histological descriptions are not nearly detailed enough-for example, the section on idiopathic inflammatory bowel disease is only 12 pages, and acute self-limited colitis is dealt with in a single paragraph. However, the major weakness is that differential diagnoses, which after all are what most of us need from such a textbook, are not even mentioned.

On the positive side, the many tables scattered throughout the book are useful and the numerous illustrations are of good size and quality. These features apart, I found little appealing about this book, even the layout of the text is rather unattractive and the cover bland, reflecting the content within. I'm sticking to the Biopsy pathology series and suggest that others do the same. 